\title{
Development of Resilient Control Systems for Technological Plants
}

\author{
Stopakevych A.O. ${ }^{1}$, Stopakevych O.A. ${ }^{2}$ \\ ${ }^{1}$ National University of Intellectual Technologies and Communications \\ ${ }^{2}$ Odessa Polytechnic National University \\ Odessa, Ukraine
}

\begin{abstract}
The aims of the research are increasing resiliency of industrial process control systems, the development of methods of creating resilient industrial process control systems and creating a resilient control system of the distillation column for the production of food alcohol. The set of objectives were achieved without the use of hardware backup, but using the developed algorithms which were based on the use of the model of dynamics of technological plants in the software of the control system and modern theory of multivariable automatic control systems. The optimal linear-quadratic controller with disturbances model and a state observer was used as a full controller. The most important result was improvement of the survivability of industrial process control systems using the developed algorithm. Also, the resilient distillation column control system was developed. For the further researches significant results are formulas which allow defining new mode values of controls in a distillation column at failure event of corresponding control output channels and formulas of logic algebra for a choice of optimum configuration of control system of a distillation column of food spirit industry. The basis of the developed technique was reconfiguration of the control system with inclusion, instead of the failed, additional devices, which were not being used in a current configuration, but were carried out the similar function taking into account dynamics coupling of a technological plant. The significance of these results is that they can be applied to the development of cyber-production systems that do not require permanent maintenance by personnel.
\end{abstract}

Keywords: resiliency, control system, linear-quadratic controller, distillation column, fault-tolerant, MIMO system, multivariable, coupled plant, optimal system.

DOI: https://doi.org/10.52254/1857-0070.2021.2-50.10

UDC: 681.51

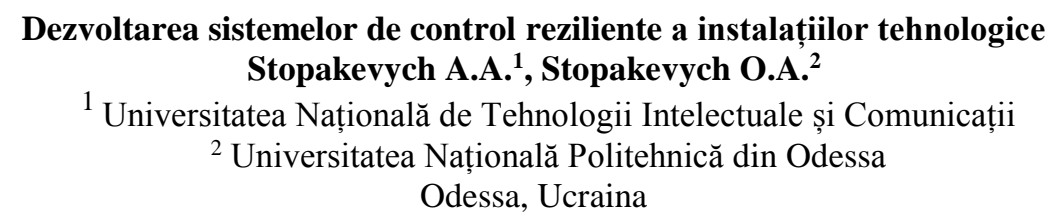

Rezumat. Obiectivele cercetării sunt creșterea resilienței sistemelor de control al instalațiilor tehnologice industriale pe baza utilizării modelului dinamic al obiectului tehnologic, dezvoltarea metodelor de creare a sistemelor de control al instalaţiilor industriale reziliente, crearea unui sistem de control rezilientr al coloanei de rectificare a producției de alcool alimentar.Obiectivele stabilite au fost atinse fără utilizarea de backup hardware iar prin intermediul algoritmilor dezvoltaţi pe baza tehnologiilor informaționale și a teoriei moderne a sistemelor de control automat multidimensional. Regulatorul liniar-cvadratic optim cu model de perturbație și observator de stare complet este utilizat ca regulator. Cel mai important rezultat este îmbunătățirea capacității de reziliență a sistemelor de control al proceselor industriale, utilizând metodologia dezvoltată pentru dezvoltarea sistemelor de control supraviețuitoare. Pentru cercetările ulterioare, rezultatele esențiale sunt formulele care permit definirea unor noi valori de mod ale cheltuielilor de control într-o coloană de rectificare la defectarea canalelor corespunzătoare de distribuție a controlului și formulele de algebră logică pentru alegerea configurației optime a sistemului de control al coloanei de rectificare a fabricării alcoolului alimentar. Baza tehnicii este reconfigurarea sistemului de control cu includerea, în locul celor refuzate, a unor dispozitive suplimentare care nu sunt utilizate în configurația actuală a sistemului de control, dar care îndeplinesc o funcție similară în vederea interrelaționării dinamicii instalației tehnologice. Semnificaţia acestor rezultate constă în faptul că ele pot fi aplicate la sarcina de a dezvolta sisteme de producție cibernetică care nu necesită întreținerea constantă a personalului tehnic.

Cuvinte cheie: rezilienţă, sistem de control, controler liniar-cvadratic, coloană de rectificare, toleranță la defecțiune, sistem MIMO, multivariabil, instalație cuplată, sistem optimizat.

(C) Стопакевич Ан. А, Стопакевич Ал. А, 2021 


\section{Разработка живучих систем управления технологическими установками \\ Стопакевич А. Ал. ${ }^{1}$, Стопакевич Ал. А. ${ }^{2}$ \\ ${ }^{1}$ Государственный университет интеллектуальных технологий и связи \\ ${ }^{2}$ Государственный университет «Одесская политехника» Одесса, Украина}

Аннотация. Целями исследования являются повышение живучести систем управления промышленными технологическими установками на основе использования в алгоритмическом обеспечении системы управления модели динамики технологического объекта, разработка методики создания живучих системы управления промышленными технологическими установками, создание живучей системы управления ректификационной колонной производства пищевого спирта. Поставленные цели были достигнуты без использования аппаратного резервирования, а за счет разработанных алгоритмов, базирующихся на информационных технологиях и современной теории многомерных систем автоматического управления. В качестве регулятора используется оптимальный линейно-квадратичный регулятор с моделью возмущений и полным наблюдателем состояния. Наиболее важным результатом является повышение живучести систем управления промышленными технологическими установками с применением разработанной методики разработки живучих систем управления. Также разработана живучая система управления ректификационной колонной. Для дальнейших исследований значимыми результатами являются формулы, которые позволяют определить новые режимные значения управляющих расходов в ректификационной колонне при отказе соответствующих каналов выдачи управления и формулы алгебры логики для выбора оптимальной конфигурации системы управления ректификационной колонной установки производства пищевого спирта. Основой методики является реконфигурация системы управления с включением, взамен отказавших, дополнительных устройств, которые не использованы в текущей конфигурации системы управления, но выполняют аналогичную функцию с учетом взаимосвязанности динамики технологической установки. Значимость полученных результатов заключается в том, что они могут быть применены для решения задачи разработки кибер-производственных систем, которые не требуют постоянного обслуживания технического персонала. При наличии поломки система управления будет продолжать удовлетворительно работать до приезда ремонтной бригады. Предложенный подход наиболее эффективен для многомерных и многосвязных систем, таких как ректификационные колонны, выпарные аппараты, сушильные установки, паровые котлы и парогенераторы, когенерационные установки.

Ключевые слова: живучесть, система управления, линейно-квадратический регулятор, ректификационная колонна, отказоустойчивость, МІМО система, многомерная, многосвязная система, оптимальная система.

\section{ВВЕДЕНИЕ}

Одной из основных тенденций развития производственно-технологических систем является переход к максимально полной автоматизации технологического оборудования, переходя в пределе к полностью автоматическим производственно-техническим комплексам. Системы подобного вида в научной литературе последних лет названы киберпроизводственными. Проблемы создания таких систем рассмотрены, например, в работах [1-3]. Отмечено, что создание киберпроизводственных систем порождает проблему повышения надежности функционирования как технологического оборудования, так и систем управления этим оборудованием. Известно, что наиболее частая причина отказов систем управления кроется в элементах, непосредственно соприкасающихся с технологическим оборудованием, то есть в датчиках и исполнительных механизмах. В последнее время ряд авторов отмечает, что причиной отказов датчиков могут быть и ки- бер-преступления [4, 5]. Управляющие контроллеры, промышленные компьютеры и полевые сети отказывают значительно реже.

Однако, отказы датчиков и исполнительных механизмов в кибер-производственной системе не должны приводить к авариям и аварийной остановке технологического оборудования. Желательно, чтобы оборудование продолжало работать, пусть и с несколько сниженным, но регламентным качеством производства и производительностью до приезда, обслуживающего производственно-техническую систему персонала, который, вообще говоря, должен находиться на аутсорсинге. Системы управления, которые обеспечивают указанные требования, называют живучими $[6,7]$.

Проблемой учета отказов в системах управления занимаются давно [8]. В процессе развития теории и практики создания живучих систем выделилось пять направлений исследований.

Первое направление является наиболее старым и связано с техническим резервировани- 
ем датчиков и исполнительных механизмов с целью повышения надежности систем [9]. При резервировании не меняется структура управляющего устройства. Такая стратегия активно применяется на особо опасных производствах, таких, например, как атомные станции. Наиболее подробно проблема внедрения систем резервирования для промышленной автоматизации рассмотрена в стандартах МЭК 61508 и 61511. Недостатком такого подхода является как высокая стоимость оборудования, так и отсутствие уверенности в используемых показателях интенсивности отказов. Так, в атомной промышленности, например, полученные результаты вероятности безотказной работы занижают в разы [10]. В свою очередь быстрое изменение номенклатуры автоматизации не позволяет получить точные опытные данные об интенсивностях отказов оборудования. Кроме того, уязвимым местом систем с резервированием являются переключающие устройства, которые, в свою очередь, также могут отказать и значительно с большей интенсивностью, чем само оборудование. Поэтому для массовых промышленных технологических процессов такой метод резервирования не целесообразен.

Второе направление является альтернативой аппаратным методам и связано с формированием множества управляющих устройств в соответствии с подключением возможных альтернативных каналов измерения и выдачи управляющих воздействий. Современный обзор основных исследований в области активных методов разработки приведен в работе [11]. Типовая система управления, сформированная в соответствии с указанным методом, имеет множество регуляторов, переключение между которыми происходит при обнаружении отказа. У систем управления такого типа само переключение может стать причиной потери устойчивости при неправильном проектировании. Пример разработки такой системы описан в работе [12]. Возможными усовершенствованиями рассмотренного метода являются метод безопасной парковки $[13,14]$, метод аналитической обратной связи [15], использование адаптивного регулятора.

Третье направление связано с использованием пассивных методов разработки отказоустойчивых систем управления. Методы отличаются тем, что разрабатывается один многомерный регулятор таким образом, чтобы при любом сочетании отказов каналов управ- ления и измерения сохранялась устойчивость замкнутой системы. Естественно, что сам объект управления при этом предполагается устойчивым. Среди работ этого направления можно отметить работу [16], где в качестве отказоустойчивого синтезируется линейноквадратический регулятор состояния, коэффициенты передачи которого могут изменяться от нуля до рассчитанного значения. При этом гарантируется многомерный запас по амплитуде. Устойчивость при отказе управлений обеспечивается соответствующим выбором весовых матриц интегрального квадратичного критерия. Недостатками подхода является то, что не рассмотрен синтез наблюдателя состояния. Кроме того, есть противоречие между надежностью и оптимальностью. Полученный регулятор как правило имеет очень небольшие коэффициенты передачи. В работе [17] решается близкая к предыдущей задача для цифрового регулятора. Задача сводится к обеспечению параметрической робастности путем выбора каналов, коэффициенты матрицы управлений для которых позволили бы обеспечить заданный интервал отклонения коэффициентов передачи. Как и в предыдущей работе вопросы устойчивости рассматриваются без наблюдателя. В работе [18] в регулятор состояния добавляется компенсатор, параметры которого определяются путем решения линейноматричного неравенства. Компенсатор поддерживает полюса замкнутой системы в заданном интервале при всех отказах управлений. Отметим, что метод работает плохо если модель объекта задана не точно.

Четвертое направление исследований связано с обобщением потенциальных возможностей стратегий активных и пассивных отказоустойчивых систем управления [19]. Важным является вывод, что чем более детализовано ставится задача, т.е. чем больше вариантов отказа разработчик пытается учесть в задаче разработки регулятора неизменной структуры, тем ниже качества переходных процессов и ниже вероятность того, что система управления справится с возмущениями, которые не были учтены в задаче синтеза. Несомненным преимуществом рассмотренных пассивных регуляторов является простота их реализации и мгновенная реакция на возмущение. Модификацией пассивных методов является использование регуляторов со скользящим режимом [20]. Но в таких регуляторах присутствуют высокочастотные виб- 
рации, что будет снижать запас устойчивости, качество переходных процессов и приводить к износу исполнительных устройств [21].

Пятым направлением является исследование систем со случайной (случайноизменяемой) структурой [22-25]. По нашему мнению, эти работы представляют скорее чисто теоретический интерес. Для адекватного расчета таких систем надо знать достоверные статистические характеристики процесса переключения структур, что практически невозможно при прогнозировании отказов.

Проведенный обзор показывает, что во всех перечисленных работах, посвященных созданию живучих систем управления, практически не рассмотрено создание таких систем для промышленных технологических установок, которые характеризуются многомерностью, многосвязностью, устойчивостью, наличием избыточных каналов измерения и, иногда, управления, довольно большой инерционностью протекающих процессов, хотя именно такие установки являются аппаратным оформлением большинства существующих технологических процессов.

Развитие современных программных технологий позволяет предложить шестое направление, связанное с проведением расчета регуляторов во время работы системы управления непосредственно в управляющем устройстве, которым является промышленный управляющий компьютер. Возможность реализовать процедуру синтеза сложных регуляторов стала широко доступной с развитием программных библиотек для языков общего назначения, с помощью которых можно решать с гарантированным результатом сложные задачи, такие как, например, задачи синтеза линейно-квадратичного многомерного оптимального регулятора. Таким образом, при отказе определенных каналов управления можно сформировать новую математическую модель динамики из моделей рабочих каналов объекта управления и рассчитать оптимальный многомерный регулятор по ней. Такую задачу назовем задачей реконфигурации системы управления. Реконфигурация - это изменение параметров и связей в системах управления без изменения цели управления. Конечно, задача реконфигурации не может быть решена полностью автоматически для произвольного объекта, но вполне может быть решена для технологических установок, процедура разработки систем управления для которых достаточно отработана, известны их статические и динамические характеристики, а также имеется успешный опыт работы систем управления, построенных на основе неточных математических моделей. Реконфигурация, как правило, ведется с использованием таблицы решений, которая строится путем анализа последствий отказов и поиска эффективной конфигурации системы управления в заданных условиях.

Задачами статьи являются создание методики разработки живучих систем управления для установок, описываемых линейными динамическими моделями высокой размерности, а также разработка живучей система управления ректификационной колонны установки производства пищевого спирта.

\section{МЕТОДИКА КОНСТРУИРОВАНИЯ ЖИВУЧЕЙ СИСТЕМЫ УПРАВЛЕНИЯ}

В соответствии с предлагаемой методикой математическая модель технологической установки должна быть задана в виде математической модели динамики. В текущей версии рассматриваются только линейные (линеаризуемые) модели. Кроме того, модель должна включать избыточное число каналов управления и каналов измерения, модель должна быть многомерной и многосвязной.

Для указанной модели задается типовая конфигурация системы управления, состоящая из выделенного подмножества каналов выдачи управления и каналов измерения. Для многомерного регулятора целесообразен выбор каналов управления с максимальным количеством перекрестных связей.

Кроме каналов, которые входят в типовую конфигурацию, должны обязательно присутствовать альтернативные каналы. Каждому элементу из множества каналов типовой конфигурации должно соответствовать заданное подмножество альтернативных каналов. Выделение альтернативных каналов определяется требованиями технологической или технико-экономической целесообразности.

Для того, чтобы задача синтеза многомерного регулятора решалась универсально, имеющуюся математическую модель динамики необходимо модифицировать. Все управляемые переменные и управляющие воздействия должны иметь близкий диапазон изменения. Наиболее целесообразно эти воздействия масштабировать таким образом, чтобы они изменялись в процентах от заданного диапазона. 
Модель многомерного управляемого и наблюдаемого объекта управления в дискретном времени зададим в виде:

$$
\begin{aligned}
& x_{i+1}=A \cdot x_{i}+B \cdot\left(u_{i}+f_{i}\right), \\
& y_{i}=C \cdot x_{i},
\end{aligned}
$$

где: $A, B, C$ - матрицы параметров объекта управления, $x_{i}$ - вектор состояния объекта $x_{i} \in \mathbb{R}^{n}, y_{i}$ - вектор измеряемых выходов $y_{i} \in \mathbb{R}^{m}, u_{i}$ - вектор управляющих воздействий $u_{i} \in \mathbb{R}^{m}, f_{i}$ - вектор приведенных к управлению возмущающих воздействий $f_{i} \in \mathbb{R}^{m}, i$ - номер шага расчета, связанный с текущим временем $t$ зависимостью $t=i \cdot \Delta t$, где $\Delta t$ - шаг дискретизации шкалы времени.

Критерий качества процессов управления в системе, в соответствии с которым будет синтезирован оптимальный многомерный линейно-квадратический регулятор, примем в виде:

$$
J=\sum_{i=0}^{\infty} x_{i}^{T} \cdot C^{T} \cdot C \cdot x_{i}+\rho \cdot u_{i}^{T} \cdot u_{i}
$$

где: $\rho>0$ - весовой коэффициент, с помощью которого настраивается качество процессов в системе.

Весовой коэффициент выбирается эмпирически с учетом желаемого качества переходных процессов в системе управления.

При функционировании систем управления технологическими установками наиболее частыми являются поломки датчиков и исполнительных устройств, что делает основную конфигурацию не работоспособной. Поломка датчика диагностируется по физической потере сигнала; показанию, которое выходит за пределы диапазона измерения или диапазона изменения параметра технологического процесса; неадекватной скорости изменения показаний. Под поломкой исполнительного механизма будем понимать то, что он остановился в некотором фиксированном положении. В дальнейшем будем считать он остановился в положении от 40 до 100\% открытия.

Фиксации отказа датчика или исполнительного механизма, которые формируют текущую конфигурацию системы управления, требует реконфигурации такой системы. Реконфигурация ведется с помощью блока вы-

бора конфигурации. Блок выбора конфигурации системы строится на основе таблицы решений, агрегирующей сигналы о работоспособности исполнительных механизмов и датчиков, и выдающей требуемую конфигурацию системы управления.

Таблица решений построена следующим образом. В первых столбцах указано, какие элементы системы управления отказали. Далее показано, какие элементы системы управления должны быть включены в данную конфигурацию с учетом отказавших элементов. В следующих столбцах указываются общие диагностические признаки, такие, например, как ожидается ли при реконфигурации падение производительности установки, снижение качества управления и т.п. В последних столбцах указана реакция на общие диагностические признаки, например, пересчет номинальных значений управляющих воздействий. Поскольку отказ исполнительного механизма влияет на процесс постоянно, то такой отказ может приводить к необходимости пересчета режимных значений управляющих расходов и, возможно, значений заданий по управляемым переменным, включая изменение производительности установки. На это указывают общие диагностические признаки. Обычно, в таблице не рассматриваются маловероятные ситуации, когда, например, отказывают два или более исполнительных механизмов, или отказывает множество датчиков. При диагностировании таких событий система управления переводится в режим ручного управления или аварийного отключения установки. Полученные на основе таблицы решений выражения алгебры логики можно минимизировать с использованием метода Куйана-МакКласки. Алгоритм минимизации описан в работе [26].

Получив новую конфигурацию системы управления, новые режимные значения управляющих переменных и заданий, ограничения управляемых переменных, произведем синтез многомерного оптимального линейно-квадратического регулятора.

Решение матричного уравнения Риккати обозначим в виде процедуры dlqr:

$$
\begin{gathered}
\operatorname{dlqr}\left(A, B, C^{T} \cdot C, \rho \cdot I\right)= \\
=\left(B^{T} \cdot P \cdot B+\rho \cdot I\right)^{-1} \cdot B^{T} \cdot P \cdot A, \\
A^{T} \cdot P \cdot A-P-A^{T} \cdot P \cdot B . \\
\cdot\left(\rho \cdot I+B^{T} \cdot P \cdot B\right)^{-1} \cdot B^{T} \cdot P \cdot A+C^{T} \cdot C .
\end{gathered}
$$


Тогда расчет полного регулятора с наблюдателем состояния и моделью возмущений в виде скачка опишем следующим алгоритмом.

1. Сформируем расширенную модель системы, которая включает последовательно соединенные модели объекта управления и возмущений в виде скачка:

$$
A_{1}=\left[\begin{array}{ll}
A & 0 \\
C & I
\end{array}\right], B_{1}=\left[\begin{array}{l}
B \\
0
\end{array}\right], C_{1}=\left[\begin{array}{ll}
0 & I
\end{array}\right] .
$$

2. Сформируем начальные значения весовых матриц для регулятора и наблюдателя:

$$
R=\rho \cdot I, Q=\left[\begin{array}{cc}
C^{T} \cdot C & 0 \\
0 & I
\end{array}\right], Q_{1}=I, R_{1}=\mu \cdot I .
$$

3. Решаем два уравнения Риккати:

$$
\begin{aligned}
& K=\left[K_{1}, K_{2}\right]=\operatorname{dlqr}\left(A_{1}, B_{1}, Q, R\right) ; \\
& L=\left[\begin{array}{c}
L_{1} \\
L_{2}
\end{array}\right]=\operatorname{dlqr}\left(A^{T}, C^{T}, Q_{1}, R_{1}\right)^{T}
\end{aligned}
$$

После расчета регулятор должен формировать следующий закон управления:

$$
\begin{aligned}
& x_{i+1}=A_{R} \cdot x_{i}+B_{R} \cdot\left(r-y_{i}\right), \\
& u_{i}=C_{R} \cdot x_{i},
\end{aligned}
$$

где:

$$
\begin{aligned}
A_{R} & =\left[\begin{array}{ccc}
A-B \cdot K_{1} & -B \cdot K_{2}-L_{1} & L_{1} \\
C & I-L_{2} & L_{2} \\
0 & 0 & I
\end{array}\right], B_{R}=\left[\begin{array}{l}
0 \\
0 \\
I
\end{array}\right], \\
C_{R} & =\left[\begin{array}{ccc}
K_{1} & K_{2} & 0
\end{array}\right],
\end{aligned}
$$

$K_{l}$ - матрица настроек пропорциональной части регулятора состояния, $K_{2}$ - матрица настроек интегральной части регулятора состояния, $L_{1}$ - матрица настроек пропорциональной части наблюдателя, $L_{2}$ - матрица настроек интегральной части наблюдателя, $r$ - вектор заданий.

\section{РАЗРАБОТКА ЖИВУЧЕЙ СИСТЕМЫ УПРАВЛЕНИЯ РЕКТИФИКАЦИОННОЙ КОЛОННОЙ}

Применим разработанную методику для создания системы управления ректификацией в производстве пищевого спирта. Типовая установка производства пищевого спирта со- стоит из трех ректификационных колонн, связанных по основному потоку. В качестве объекта управления возьмем первую, бражную, колонну установки производительностью 60 тонн спирта в сутки.

Максимальное число управляющих воздействий для колонны равно трем. Это изменения расхода бражки $u_{1}$, расхода пара $u_{2}$ и расхода охлаждающей воды дефлегматора $u_{3}$. Размерностью всех управлений является процент хода исполнительного механизма. Основными возмущения является отклонение концентрации спирта в бражке, давления пара в паропроводе, температуры охлаждающей воды. Для удобства моделирования указанные возмущения приводятся к каналам выдачи управлений с учетом статических взаимосвязей. Набор измеряемых переменных включает: $y_{1}$ - давление снизу колонны, Па; $y_{2}$ - давление вверху колонны, Па; $y_{3}-$ температура снизу колонны, ${ }^{\circ} \mathrm{C} ; y_{4}-$ температура вверху колонны, ${ }^{\circ} \mathrm{C} ; y_{5}-$ температура бражки после дефлегматора, ${ }^{\circ} \mathrm{C} ; y_{6}-$ температура воды после дефлегматора, ${ }^{\circ} \mathrm{C}$. Отметим, что в исследовании [27] была получена связь качественного параметра $Y$ (концентрация спирта в паровом потоке) с легко измеряемой косвенной величиной $y_{4}$. Полученная зависимость имеет вид:

$$
Y=548-5.37 \cdot y_{4} \text {. }
$$

Поэтому концентрацию $Y$ в конфигурацию системы управления включать нецелесообразно. Однако, точная корреляция проявляется в случае, когда значение температуры не отклоняется от номинала более половины

\begin{tabular}{|c|c|c|c|c|c|c|c|c|}
\hline \multirow{5}{*}{ 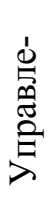 } & & \multicolumn{6}{|c|}{ Измерения / Measurements } & \multirow{5}{*}{ 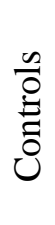 } \\
\hline & & $y_{I}$ & $y_{2}$ & $y_{3}$ & $y_{4}$ & $y_{5}$ & $y_{6}$ & \\
\hline & $u_{1}$ & + & + & & + & + & + & \\
\hline & $u_{2}$ & + & + & + & + & + & + & \\
\hline & $u_{3}$ & + & + & + & + & + & + & \\
\hline
\end{tabular}
градуса. При плохо спроектированной системе управления соблюдение температуры с такой точностью является проблемным. Матрица взаимосвязей каналов управления колонны приведена в табл. 1.

Таблица $1^{1}$. Матрица взаимосвязей колонны как объекта управления ${ }^{2}$ 
При условии, что система управления может поддерживать задания, если число управляемых переменных равно числу управляющих воздействий, приходим к выводу, что на каждое управляющее воздействие приходится две управляемые переменные.

Экспериментальная математическая модель колонны, имеет вид, приведенный в табл. 2 [27].

Таблица $2^{3}$.

Матрица передаточных функций колонны ${ }^{4}$

\begin{tabular}{|c|c|c|c|}
\hline № & $u_{1}$ & $u_{2}$ & $u_{3}$ \\
\hline$y_{1}$ & $\frac{-49}{500 s+1} e^{-30 s}$ & $\frac{239}{66 s+1}$ & $\frac{-10}{90 s+1}$ \\
\hline$y_{2}$ & $\frac{-173}{370 s+1}$ & $\frac{287}{73 s+1}$ & $\frac{-10.8}{220 s+1}$ \\
\hline$y_{3}$ & - & $\frac{0.025}{120 s+1} e^{-30 s}$ & $\frac{-0.03}{340 s+1} e^{-200 s}$ \\
\hline$y_{4}$ & $\frac{-0.15}{450 s+1} e^{-72 s}$ & $\frac{0.05}{220 s+1} e^{-60 s}$ & $\frac{-0.009}{190 s+1} e^{-100 s}$ \\
\hline$y_{5}$ & $\frac{-0.22}{435 s+1} e^{-180 s}$ & $\frac{0.22}{800 s+1} e^{-240 s}$ & $\frac{-0.13}{360 s+1} e^{-220 s}$ \\
\hline$y_{6}$ & $\frac{0.9}{423 s+1} e^{-160 s}$ & $\frac{0.1}{200 s+1} e^{-150 s}$ & $\frac{-0.285}{268 s+1} e^{-74 s}$ \\
\hline
\end{tabular}

Bсе временные параметры в матрице приведены в секундах.

Для унификации вклада переменных в интегральный квадратический критерий качества, измеряемые переменные перемасштабируем (см. табл. 3), применив соответствующие пересчетные коэффициенты, приведенные в табл. 4.

Таблица $3^{5}$.

Нормализованная матрица передаточных функций $^{6}$

\begin{tabular}{|c|c|c|c|}
\hline № & $u_{1}$ & $u_{2}$ & $u_{3}$ \\
\hline$y_{1}$ & $\frac{-1.03}{8.33 s+1} e^{-0.5 s}$ & $\frac{5}{1.1 s+1}$ & $\frac{-0.21}{1.5 s+1}$ \\
\hline$y_{2}$ & $\frac{-3.01}{6.17 s+1}$ & $\frac{5}{1.22 s+1}$ & $\frac{-0.19}{3.67 s+1}$ \\
\hline$y_{3}$ & - & $\frac{4.17}{2 s+1} e^{-0.5 s}$ & $\frac{-5}{5.67 s+1} e^{-3.33 s}$ \\
\hline$y_{4}$ & $\frac{-5}{7.5 s+1} e^{-1.2 s}$ & $\frac{1.67}{3.67 s+1} e^{-1.0 s}$ & $\frac{-0.3}{3.17 s+1} e^{-1.67 s}$ \\
\hline$y_{5}$ & $\frac{-5}{7.25 s+1} e^{-3 s}$ & $\frac{5}{13.33 s+1} e^{-4 s}$ & $\frac{-2.95}{6 s+1} e^{-3.67 s}$ \\
\hline$y_{6}$ & $\frac{5}{7.05 s+1} e^{-2.67 s}$ & $\frac{0.56}{3.33 s+1} e^{-2.5 s}$ & $\frac{-1.58}{4.47 s+1} e^{-1.23 s}$ \\
\hline
\end{tabular}

Кроме того, для удобства компьютерного моделирования переведем временные постоянные в минуты.

Таблица решений для реконфигурации живучей системы управления имеет вид, приведенный в табл. 5. Обозначения, которые приведены в таблице и формулах: буква $o$ перед обозначением параметра означает признак неработоспособности, буква $v$ - признак включения в конфигурацию, $p r$ - сигнал об изменении производительности колонны, q - сигнал об изменении производительности колонны, $a f$ - сигнал о пересчете номинала $u_{1}$ на основе предыдущего значения $u_{2}, a v$ - сигнал о пересчете номинала $u_{2}$ на основе предыдущего значения $u_{1}, i m$ - сигнал для перехода в ручной режим управления.

Таблица 4 . Нормализующие множители для параметров модели ${ }^{8}$

\begin{tabular}{|l|l|l|l|l|l|}
\hline$y_{1}$ & $y_{2}$ & $y_{3}$ & $y_{4}$ & $y_{5}$ & $y_{6}$ \\
\hline 47.8 & 57.4 & 0.006 & 0.03 & 0.044 & 0.18 \\
\hline
\end{tabular}

На основе приведенной таблицы решений получены формулы алгебры логики для определения соответствующей конфигурации системы управления. Для упрощения в формулах исключен случай, когда конфигурация регламентная. Таким образом, последующие формулы - это формулы для реконфигурации.

Включение управляющих механизмов определяются по следующим формулам:

$$
v u_{1}=\overline{o u_{1}}, v u_{2}=\overline{o u_{2}}, \quad v u_{3}=\overline{o u_{3}}
$$

Включение датчиков определяется по следующим формулам:

$$
\begin{gathered}
v y_{1}=\left(o y_{6} \wedge \overline{o y_{4}}\right) \vee\left(o y_{2} \wedge o y_{4}\right) \vee \ldots \\
\ldots \vee\left(o y_{2} \wedge o y_{6}\right) \vee o y_{2} \vee\left(\overline{o y_{6}} \wedge o y_{4}\right) \\
v y_{2}=o u_{1} \vee o u_{2} \vee o u_{3} \vee\left(o y_{4} \wedge o y_{6}\right) \\
v y_{3}=o y_{4} \wedge o y_{6} \\
v y_{4}=o u_{1} \vee o u_{2} \vee o u_{3} \vee\left(\overline{o y_{4}} \wedge\left(o y_{6} \vee o y_{2}\right)\right) \\
v y_{5}=o y_{4} \vee o y_{6} \\
v y_{6}=\overline{o y_{6}} \wedge\left(o y_{2} \vee o y_{4}\right)
\end{gathered}
$$

Необходимость пересчета и стабилизации в новом режиме работы колонны определяется по следующим формулам:

$$
\begin{array}{r}
a f=o u_{2}(16) \\
a v=o u_{1}(17) \\
p r=o u_{1} \vee o u_{2}(18) \\
q=o u_{1} \vee o u_{2} \vee o u_{3}(19)
\end{array}
$$


Таблица 5 .

Таблица решений для реконфигурации живучей системы управления ${ }^{10}$

\begin{tabular}{|c|c|c|c|c|c|c|c|c|c|c|c|c|c|c|c|c|c|c|c|}
\hline \multirow{3}{*}{ 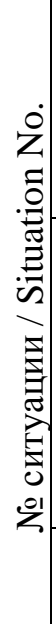 } & \multicolumn{6}{|c|}{ Условия / Conditions } & \multicolumn{11}{|c|}{$\begin{array}{c}\text { Реконфигурация системы управления и } \\
\text { пересчет многомерного регулятора / } \\
\text { Control system reconfiguration changing and } \\
\text { MIMO controller redesign }\end{array}$} & \multicolumn{2}{|c|}{$\begin{array}{c}\text { Ожидаемые } \\
\text { результаты } \\
\text { действий / } \\
\text { Expected outcomes }\end{array}$} \\
\hline & \multicolumn{6}{|c|}{$\begin{array}{c}\text { Что отказало в } \\
\text { регламентной } \\
\text { конфигурации / What } \\
\text { is failed in standard } \\
\text { control configuration }\end{array}$} & \multicolumn{9}{|c|}{$\begin{array}{c}\text { Что включено в аварийную } \\
\text { конфигурацию / What is included } \\
\text { is alarm configuration }\end{array}$} & \multicolumn{2}{|c|}{$\begin{array}{l}\text { Перес } \\
\text { чет / } \\
\text { Recal- } \\
\text { cula- } \\
\text { tion }\end{array}$} & $\begin{array}{l}\text { Паде- } \\
\text { ние } \\
\text { качест- } \\
\text { ва/ } \\
\text { Quality } \\
\text { loss }\end{array}$ & $\begin{array}{l}\text { Падение } \\
\text { произ- } \\
\text { водите- } \\
\text { льности/ } \\
\text { Producti- } \\
\text { vity loss }\end{array}$ \\
\hline & $\Xi$ & 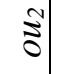 & $\stackrel{\Xi}{\Xi}$ & $\tilde{\partial}$ & $\partial^{+}$ & ठे & $\Xi$ & 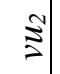 & $\stackrel{3}{\Xi}$ & సี & $\stackrel{+}{د}$ & $\stackrel{b}{2}$ & $\bar{\lambda}$ & $\stackrel{\check{z}}{\overrightarrow{2}}$ & $\sum^{2}$ & 3 & పे & & \\
\hline 1 & 0 & 0 & 0 & 0 & 0 & 0 & 1 & 1 & 1 & 1 & 1 & 1 & 0 & 0 & 0 & 0 & 0 & 0 & 0 \\
\hline 2 & 1 & 0 & 0 & 0 & 0 & 0 & 0 & 1 & 1 & 1 & 1 & 0 & 0 & 0 & 0 & 0 & 1 & 0 & 1 \\
\hline 3 & 0 & 1 & 0 & 0 & 0 & 0 & 1 & 1 & 0 & 1 & 1 & 0 & 0 & 0 & 0 & 1 & 0 & 0 & 1 \\
\hline 4 & 0 & 0 & 1 & 0 & 0 & 0 & 1 & 0 & 1 & 1 & 1 & 0 & 0 & 0 & 0 & 1 & 1 & 0 & 1 \\
\hline 5 & 0 & 0 & 0 & 1 & 0 & 0 & 1 & 1 & 1 & 0 & 1 & 1 & 1 & 0 & 0 & 0 & 0 & 1 & 0 \\
\hline 6 & 0 & 0 & 0 & 0 & 1 & 0 & 1 & 1 & 1 & 0 & 0 & 1 & 1 & 1 & 0 & 0 & 0 & 1 & 0 \\
\hline 7 & 0 & 0 & 0 & 0 & 0 & 1 & 1 & 1 & 1 & 0 & 1 & 0 & 1 & 1 & 0 & 0 & 0 & 1 & 0 \\
\hline 8 & 0 & 0 & 0 & 1 & 1 & 0 & 1 & 1 & 1 & 0 & 0 & 1 & 1 & 1 & 0 & 0 & 0 & 1 & 0 \\
\hline 9 & 0 & 0 & 0 & 0 & 1 & 1 & 1 & 1 & 1 & 1 & 0 & 0 & 0 & 1 & 1 & 0 & 0 & 1 & 0 \\
\hline 10 & 0 & 0 & 0 & 1 & 0 & 1 & 1 & 1 & 1 & 0 & 0 & 1 & 1 & 1 & 0 & 0 & 0 & 1 & 0 \\
\hline 11 & Дру & $\begin{aligned} \text { гие } \\
\text { ртка } \\
\mathrm{O} \\
\text { figu }\end{aligned}$ & $\begin{array}{l}\text { кон } \\
30 \mathrm{O}, \\
\text { ther } \\
\text { ratic }\end{array}$ & & $1 /$ & & & $\begin{array}{l}\text { Пер } \\
\text { Swi }\end{array}$ & tch & & yП & $\begin{array}{l}\text { вки } \\
\text { равл } \\
\text { уste }\end{array}$ & & mant & & & & $\begin{array}{r}3 a \\
p \\
\text { yחr } \\
\text { Dep } \\
\text { manu }\end{array}$ & $\begin{array}{l}\text { вят от } \\
\text { ного } \\
\text { тения / } \\
\text { s on the } \\
\text { speration }\end{array}$ \\
\hline
\end{tabular}

Как было сказано выше, в таблице 5 присутствуют признаки $a f$ и $a v$.

Эти признаки запускают при отказе процедуру перевода управлений в рациональное для данной конфигурации номинальное значение соответствующего расхода.

Критерием рациональности для бражной колонны является соотношение $F / V$ для получения бражного дистиллята заданной концентрации при известной концентрации спирта в бражке с коэффициента избытка паpa 1.05 .

Формулы расчета (20), (21) получены нами в результате аппроксимации номограммы, приведенной в работе [27]. Коэффициенты формул (20), (21) приведены в табл. 6. Особенностью формул является то, что учет нелинейных эффектов взаимодействия параметров, потребовал для достижения необходимой точности аппроксимации использова- ния уравнение высокого (четвертого) порядка. .

Таблица $6^{11}$.

Коэффициенты формул (20) и (21) ${ }^{12}$

\begin{tabular}{|l|r|l|r|}
\hline$a_{1}$ & +7.86345000 & $b_{1}$ & $+5.15183 \cdot 10^{+2}$ \\
\hline$a_{2}$ & $-4.69949 \cdot 10^{+1}$ & $b_{2}$ & $-2.80121 \cdot 10^{+2}$ \\
\hline$a_{3}$ & $+2.62048 \cdot 10^{-4}$ & $b_{3}$ & $-1.37755 \cdot 10^{-2}$ \\
\hline$a_{4}$ & +3.10543000 & $b_{4}$ & $-2.95763 \cdot 10^{+1}$ \\
\hline$a_{5}$ & $-2.20122 \cdot 10^{-2}$ & $b_{5}$ & +1.331530000 \\
\hline$a_{6}$ & $-5.58864 \cdot 10^{-2}$ & $b_{6}$ & $+5.16582 \cdot 10^{-1}$ \\
\hline$a_{7}$ & $-7.40633 \cdot 10^{-6}$ & $b_{7}$ & $-7.77778 \cdot 10^{-3}$ \\
\hline$a_{8}$ & $+3.03876 \cdot 10^{-4}$ & $b_{8}$ & $-2.82738 \cdot 10^{-3}$ \\
\hline$a_{9}$ & -1.340890000 & $b_{9}$ & $+1.13613 \cdot 10^{+1}$ \\
\hline$a_{10}$ & $+3.33698 \cdot 10^{-2}$ & $b_{10}$ & $-1.93676 \cdot 10^{-1}$ \\
\hline$a_{11}$ & $+1.33362 \cdot 10^{-1}$ & $b_{11}$ & $-2.38187 \cdot 10^{+1}$ \\
\hline$a_{12}$ & $-2.43400 \cdot 10^{-4}$ & $b_{12}$ & $+1.19048 \cdot 10^{-3}$ \\
\hline$a_{13}$ & $+7.01173 \cdot 10^{-3}$ & $b_{13}$ & $-5.82222 \cdot 10^{-1}$ \\
\hline$a_{14}$ & $-5.06914 \cdot 10^{-5}$ & $b_{14}$ & $+3.87879 \cdot 10^{-2}$ \\
\hline$a_{15}$ & $+2.60416 \cdot 10^{+2}$ & $b_{15}$ & $+2.41877 \cdot 10^{+3}$ \\
\hline
\end{tabular}


Полученные нами формулы имеют вид:

$$
\begin{aligned}
& V=a_{1} \cdot Y+a_{2} \cdot F+a_{3} \cdot Y^{2} \cdot F^{2}+\ldots \\
& \ldots+a_{4} \cdot Y \cdot F+a_{5} \cdot Y \cdot F^{2}+\ldots \\
& \ldots+a_{6} \cdot Y^{2} \cdot F+a_{7} \cdot Y \cdot F^{3}+\ldots \\
& \ldots+a_{8} \cdot Y^{3} \cdot F+a_{9} \cdot Y^{2}+a_{10} \cdot Y^{3}+\ldots \\
& \ldots+a_{11} \cdot F^{2}+a_{12} \cdot Y^{4}+a_{13} \cdot F^{3}+ \\
& \ldots+a_{14} \cdot F^{4}+a_{15} \\
& F=b_{1} \cdot V+b_{2} \cdot Y+b_{3} \cdot Y^{2} \cdot V^{2}+ \\
& \ldots+b_{4} \cdot Y \cdot V+b_{5} \cdot Y \cdot V^{2}+\ldots \\
& \ldots+b_{6} \cdot Y^{2} \cdot V+b_{7} \cdot Y \cdot V^{3}+\ldots \\
& \ldots+b_{8} \cdot Y^{3} \cdot V+b_{9} \cdot Y^{2}+\ldots \\
& \ldots+b_{10} \cdot Y^{3}+b_{11} \cdot V^{2}+ \\
& \ldots+b_{12} \cdot Y^{4}+b_{13} \cdot V^{3}+. b_{14} \cdot V^{4}+b_{15} .
\end{aligned}
$$

где: $Y$ - регламентная концентрация спирта в паре (объемные \%); $F$ - регламентный расход бражки, кг/с; $V$ - регламентный расход пара, кг/с.

При пересчете регламентных расходов следует пересчитать и регламентный расход охлаждающей воды $(G$, кг/с), однако это целесообразно только при условии, что вода имеет приблизительно стабильную температуру:

$$
G=((F / V) \cdot 1.61)
$$

Таким образом, упрощенная программнотехническая структура разработанной системы управления может быть представлена на рис. 1.

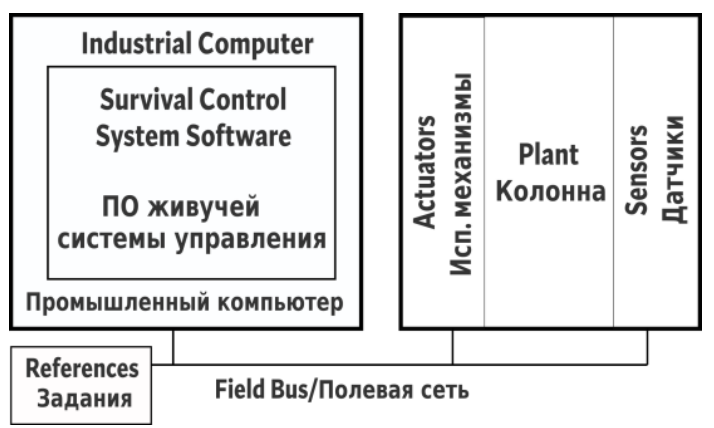

Рис. 1. Упрощенная программно-техническая структура разработанной системы управления ${ }^{13}$.

Поскольку в технической структуре используется полевая компьютерная сеть, то все датчики и исполнительные механизмы могут иметь внутреннюю систему самодиагностики с выставлением признака отказа, что упрощает решение задачи реконфигурации.

Схема алгоритма функционирования живучей системы управления ректификационной колонной производства пищевого спирта в реальном времени представлена на рис. 2 (начальное значение $M=0$ ).

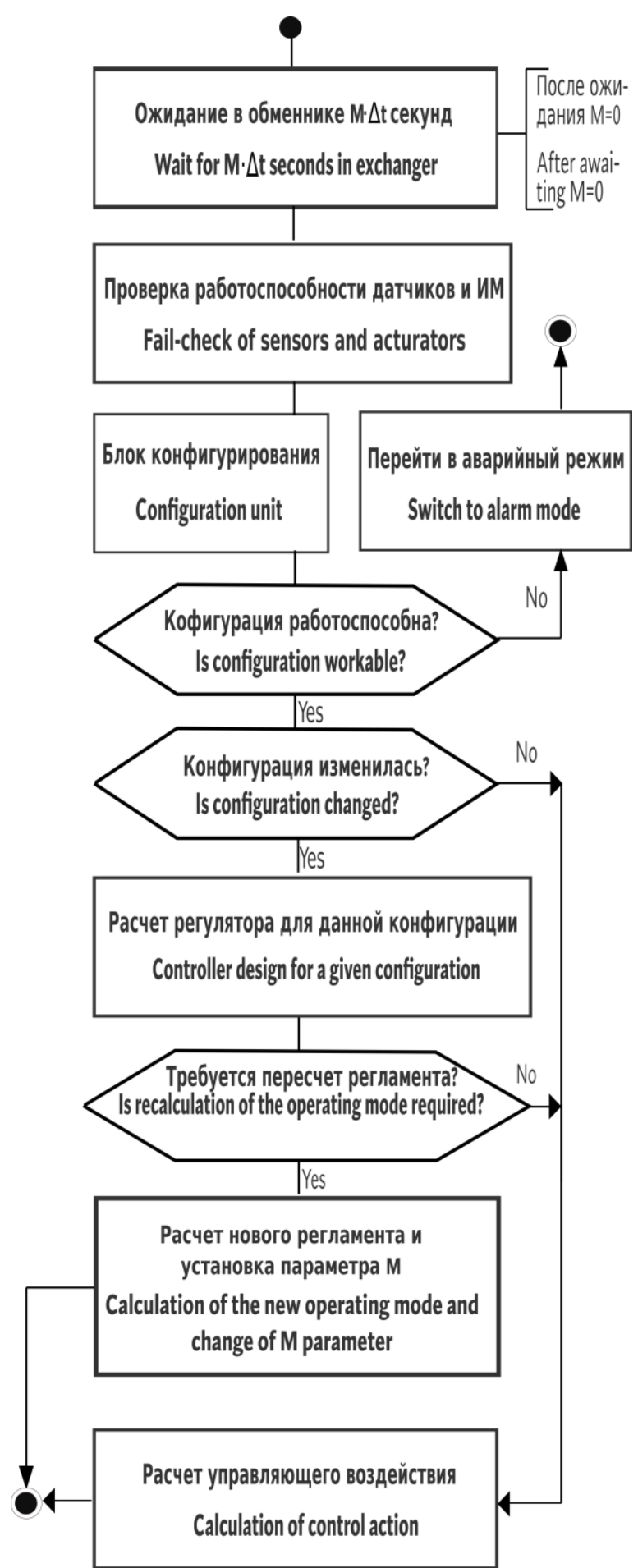

Рис. 2. Схема алгоритма функционирования живучей системы управления ${ }^{14}$. 
Для проверки качества разработанной живучей системы и применимости разработанной методики к реальным технологическим объектам создана имитационная модель, исследование которой проведем в следующем разделе.

\section{ИМИТАЦИОННОЕ МОДЕЛИРОВА- НИЕ ЖИВУЧЕЙ СИСТЕМЫ УПРАВЛЕ- НИЯ РЕКТИФИКАЦИОННОЙ КОЛОН- НОЙ}

Схема аналогово-дискретной имитационной модели живучей системы управления ректификационной колонны установки производства пищевого спирта показана на рис. 3.

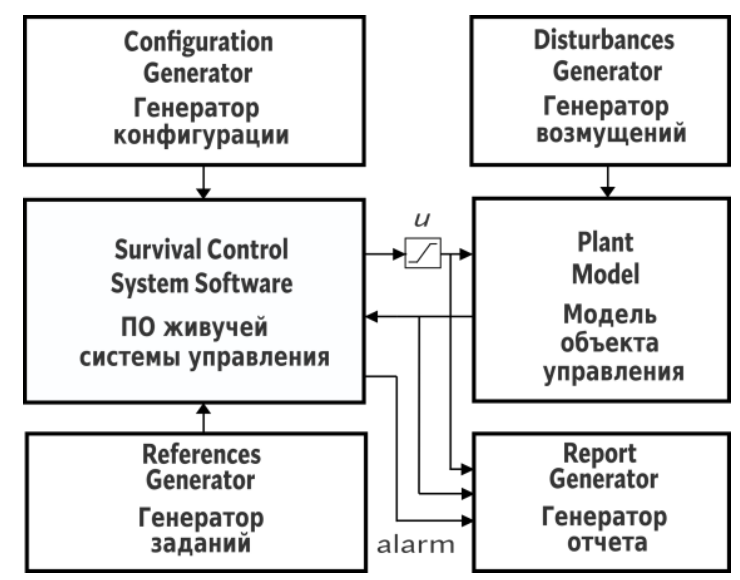

Рис. 3. Схема имитационной модели живучей системы управления ${ }^{15}$.
Модель объекта управления функционирует в аналоговом времени, а остальные подсистемы - в дискретном времени. Генераторы в соответствии с временным регламентом имитируют тестовые технологические события отказа оборудования и подачи сильных скачкообразных возмущающих воздействий в течение заданного интервала времени.

Всего исследование было проведено для семи событий, которые являются наиболее характерными (рис. 4). Качество функционирования системы управления оценивалось по работоспособности сформированной конфигурации в заданной технологической ситуации и качеству переходных процессов (максимальное отклонение и время установления). Для того, чтобы приблизить имитационное моделирование к реальности, величины управляющих воздействий ограничивались, а возмущения выбирались исходя из экспериментально зафиксированных максимальных для рассматриваемого объекта значений.

Во всех рассмотренных случаях система управления не вышла за регламентные границы технологических переменных, даже если датчики, которые их измеряли, отказывали (табл. 7). Таким образом, разработанная система управления является пригодной для использования.

Максимальные отклонения переменных, полученные в результате имитационного

Таблица $7^{16}$. моделирования ${ }^{17}$

\begin{tabular}{|c|c|c|c|c|c|c|c|c|c|c|}
\hline № & $\begin{array}{c}\text { Событие/ } \\
\text { Event }\end{array}$ & $y_{1}$ & $y_{2}$ & $y_{3}$ & $y_{4}$ & $y_{5}$ & $y_{6}$ & $u_{1}$ & $u_{2}$ & $u_{3}$ \\
\hline I-II & $f_{l}=3$ & 117.11 & 104.02 & 106.01 & 94.88 & 79.8 & 55.78 & 73 & 70.5 & 69.2 \\
\hline III-IV & $f_{l}=0$ & 116.88 & 103.97 & 105.98 & 95.12 & 80.17 & 54.19 & 66.9 & 69.37 & 70.85 \\
\hline IV-V & $o y_{4}=1$ & \multicolumn{9}{|c|}{ Без значимых отклонений / Without significant deviations } \\
\hline $\mathrm{V}-\mathrm{VI}$ & $f_{l}=3$ & 115.4 & 102.01 & 106.43 & 95.15 & 81.72 & 59.37 & 69.98 & 86.26 & 50.5 \\
\hline VI-VII & $\begin{array}{l}o u_{1}=1 \\
f_{2}=-5\end{array}$ & 117.05 & 103.88 & 106.09 & 94.84 & 80.4 & 54.78 & 66.64 & 89 & 64.68 \\
\hline VII-VIII & $\begin{array}{c}f_{1}=0 \\
f_{2}=0 \\
o u_{1}=1\end{array}$ & 117.34 & 104.32 & 106.05 & 94.78 & 79.56 & 56.32 & 75 & 72 & 75 \\
\hline VIII-IX & $\begin{array}{c}o y_{2}=1 \\
o y_{6}=1 \\
f_{l=} f_{2=} f_{3}=5\end{array}$ & 117.02 & 104.01 & 105.99 & 95.08 & 79.93 & 55.09 & 60 & 65 & 65 \\
\hline \multicolumn{2}{|c|}{$\begin{array}{c}\text { Регламент / } \\
\text { Operation mode }\end{array}$} & 117 & 104 & 106 & 95 & 80 & 55 & 70 & 70 & 70 \\
\hline
\end{tabular}




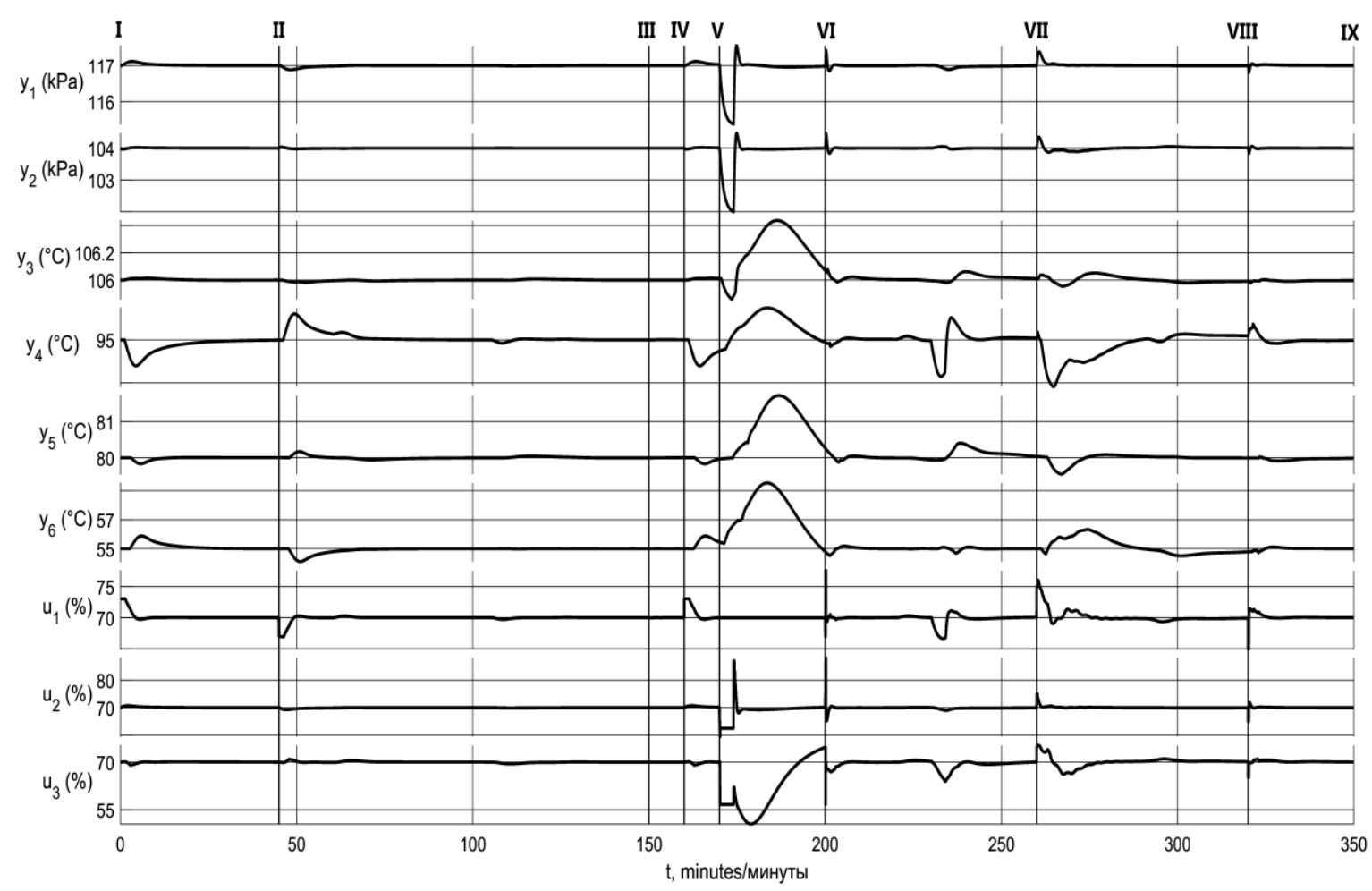

Рис. 4. Результаты имитационного моделирования живучей системы управления ректификационной колонны ${ }^{18}$.

\section{ВЫВОДЫ}

Первым результатом работы является создание методики создания живучих систем управления сложными технологическими установками. Базой методики является задача синтеза линейно-квадратического многомерного оптимального регулятора, которая формируется и решается так, чтобы она могла быть решена с одинаковым качеством для любой многомерной, линеаризованной устойчивой управляемой и наблюдаемой математической модели динамики объекта управления. Для этого математическая модель объекта управления специальным образом нормализуется. Новый регулятор рассчитывается непосредственно в программном обеспечении системы управления, что гарантирует устойчивость и работоспособность полученной системы управления. Последняя обеспечивается путем реализации в системе управления процедуры установления в новом режиме при отказе исполнительных механизмов. Номинальные значения управляющий воздействий пересчитываются исходя из того, что одно из управляющих воздействий постоянно.

Вторым результатом работы является создание живучей системы управления ректи- фикационной колонной производства пищевого спирта на основе применения разработанной методики.

Выбранная для исследований бражная колонна является наиболее энергоемким и во многом определяющим качество конечного продукта аппаратом установки производства пищевого спирта. Анализ матрицы взаимосвязей колонны показывает, что объект имеет три входные переменные и шесть выходных переменных, которые могут быть использованы для управления. Кроме того, объект может ограниченно функционировать при отказе управляющих воздействий. Эти особенности могут быть использованы для создания разных конфигураций систем управления. Разработаны правила реконфигурации живучей системы управления колонны в таблицы решений и совокупности логических правил. Получены уравнения, позволяющие пересчитывать номинальные значения управляющих расходов при отказах, а также изменять производительность колонны. Для проверки работы созданной живучей системы управления проведен имитационный эксперимент, который показал высокое качество функционирование живучей системы управления. 


\section{APPENDIX 1 (ПРИЛОЖЕНИЕ 1)}

${ }^{1,2}$ Table 1. Column interconnection matrix.

3,4 Table 2. Column transfer function matrix.

5,6 Table 3. Normalized transfer function matrix.

${ }^{7,8}$ Table 4. Normalizing factors for model parameters.

${ }^{9,10}$ Table 5. Table of rules for reconfiguration of the survivable control system.

11,12 Table 6. Coefficients of (18), (19) equations.

${ }^{16,17}$ Table 7. Maximum deviations of variables obtained from simulation.

${ }^{13}$ Fig. 1. Simplified software and hardware structure of the developed control system.

${ }^{14}$ Fig. 2. Diagram of functioning algorithm of the survivable control system.

${ }^{15}$ Fig. 3. Scheme of simulation model of the survivable control system.

${ }^{18}$ Fig. 4. Results of simulation of the survivable control system of a distillation column.

\section{Литература (References)}

[1] Meissner H., Aurich J. Implications of CyberPhysical Production Systems on Integrated Process Planning and Scheduling. Procedia Manufacturing, 2019, vol. 28, pp. 167-173. doi: 10.1016/j.promfg.2018.12.027

[2] Ahmadi A., Cherifi C., Cheutet V., Ouzrout Y. A Review of CPS 5 Components Architecture for Manufacturing Based on Standards. 11th IEEE International Conference on Software, Knowledge, Information Management and Applications. Colombo (Sri Lanka), 2017. doi: 10.1109/SKIMA.2017.8294091

[3] Brettel M., Friederichsen N., Keller M., Rosenberg N. How Virtualization, Decentralization and Network Building Change the Manufacturing Landscape: An Industry 4.0 Perspective. International Journal of Science, Engineering and Technology, 2014, no. 8, pp. 37-44. doi: 10.1016/j.promfg.2018.12.027

[4] Ao W., Song Y., Wen C. Adaptive CyberPhysical System Atack Detection and Reconstruction with Application to Power Systems. IET Control Theory Appl, 2016, vol.10, no. 12, pp. 1458-1468. doi: 10.1049/ietcta.2015.1147.

[5] Deng R., Xiao G., Lu R. Defending Against False Data Injection Attacks on Power System State Estimation. IEEE Trans. Ind. Inform, 2017, no. 13, pp. 198-207.

[6] Knight J., Sullivan K., Elder M., Wang C. Survivability Architectures: Issues and Approaches. Proceedings DARPA Information Survivability Conference and Exposition. DISCEX'00. Hilton Head (SC, USA). 2000, vol.2, pp. 157-171, doi: 10.1109/DISCEX.2000.821517

[7] Stekol'nikov Yu.I. Zhivuchest' sistem. [Survivability of systems]. Saint-Petersburg. 2002. 155 p. (in Russian).
[8] Aviziens A. Fault-Tolerant Systems. IEEE Transactions on Computers, 1976, vol. 25, no. 12, pp. 1304-1312. doi: 10.1109/TC.1976.1674598

[9] Kashtanov V.A., Medvedev A.I. Teoriya nadezhnosti slozhnykh sistem [Reliability Theory of Complex Systems]. Moscow. 2010. 608 p. (in Russian).

[10]RB-100-15. Rekomendatsii po poryadku vypolneniya analiza nadezhnosti sistem $i$ elementov atomnykh stantsiy, vazhnykh dlya bezopasnosti, i ikh funktsiy. [Safety manual 2015. Recommendations on how to perform reliability analysis of safety important systems and elements of nuclear plants and their functions]. Moscow, Federal Service for Ecological, Technological and Nuclear Supervision, 2015. (in Russian).

[11] Abbaspour A, Mokhtari S, Sargolzaei A, Yen K.K. A Survey on Active Fault-Tolerant Control Systems. Electronics, 2020, no. 9, pp. 15131537. doi: 10.3390/electronics 9091513

[12] Yang H., Jiang B., Staroswiecki M. Supervisory Fault Tolerant Control for a Class of Uncertain Nonlinear Systems. Automatica, 2009, vol. 45, no. $10, \quad$ pp. 2319-2324. doi:10.1016/j.automatica.2009.06.019

[13] Gandhi R., Mhaskar P. A Safe-parking Framework for Plant-Wide Fault-Tolerant Control. Chemical Engineering Science, 2009, vol. 64, no. 13, pp. 3060-3071. doi: 10.1016/j.ces.2009.03.039

[14] Miao D., Rahul G., Prashant M. An Integrated Fault Detection and Isolation and Safe-Parking Framework for Networked Process Systems. Industrial \& Engineering Chemistry Research, 2011, vol. 50, no. 9, pp. 5667-5679. doi: 10.1021/ie101786t

[15] Abbaspour A., Yen K., Forouzannezhad P., Sargolzaei A. An Adaptive Resilient Control Approach for Pressure Control in Proton Exchange Membrane Fuel Cells. IEEE Transactions on Industry Applications, 2019. vol. 55, no. 6, pp. 6344 - 6354. doi: 10.1109/TIA.2019.2929256

[16] Vellette R. Reliable Linear-Quadratic StateFeedback Control. Automatica, 1995, vol. 31, no. 1, pp. 137-143 doi: 10.1016/00051098(94)E0045-J

[17] Yang Y., Yang G. Soh Y. Reliable Control of Discrete-Time Systems With Actuator Filure. IEE Proceedings - Control Theory and Applications, 2000, vol. 147, no. 4, pp. 428-432. doi: 10.1049/ip-cta:20000553

[18]Zhao Q, Jiang J. Reliable State Feedback Control System Design against Actuator Failures. Automatica, 1998, vol. 34, no. 10, pp. 1267-1272. doi: 10.1016/S00051098(98)00072-7 
[19] Jiang J., Yu X. Fault-Tolerant Control Systems: A Comparative Study Between Active and Passive Approaches. Annual Reviews in Control, 2012, vol. 36, no. 1, pp. 60-72. doi: 10.1016/j.arcontrol.2012.03.005

[20] Amin A., Hasan K. A Review of Fault Tolerant Control Systems: Advancements and Applications. Measurement, 2019, vol. 143. pp. 58-68. doi: 10.1016/j.measurement.2019.04.083

[21] Utkin V. Chattering Problem. Preprints of the 18th IFAC World Congress. Milano (Italy), 2011. pp. 13374-13379

[22]Zhuk S. Ya. Otsenivanie stokhasticheskikh protsessov so sluchaynoy strukturoy s markovskimi pereklyucheniyami $\mathrm{v}$ diskretnom vremeni (obzor). [Estimation of stochastic processes with a random structure with Markov switching in discrete time (review)]. Izvestiya vysshikh uchebnykh zavedeniy. Radioelektronika, 2020, vol. 63, no.10, pp. 591607. (in Russian). doi: 10.20535/S0021347020100015

[23] Boldinov V., Bukhalev V., Pryadkin S., Skrynnikov A. Control Algorithm for a Queuing System Based on the Theory of Systems with Random Jump Structure. J. Comput. Syst. Sci.

\section{Сведения об авторах.}

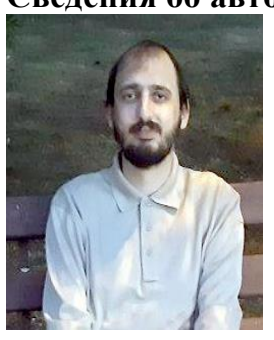

\section{Стопакевич Андрей Алексеевич,}

кандидат технических наук, доцент.

Государственный университет интеллектуальных технологий и связи.

Область научных интересов сложные системы автоматического управления технологическими процессами, программные технологии в области автоматизации производства.

Украина, Одесса

E-mail: stopakevich@gmail.com
Int., 2015, vol. 54, no.2, pp. 218-229. https://doi.org/10.1134/S1064230715010025

[24]Buravlev A., Kazakov I. A Model of the Reliability of a Self-Regenerating System With a Random Structure. Journal of Computer and Systems Sciences International, 2001, vol. 40, pp. 40-42.

[25] Bukhalyov V.A., Boldinov V.A., Skrynnikov A.A. Upravlenie sluchaynoy skachkoobraznoy strukturoy ob"ekta v usloviyakh protivodeystviya [Control of random jump structure of an object under counteracting conditions]. XIII Vserossiyskoe soveshchanie po problemam upravleniya VSPU-2019 [Proc. of XIII All-Russian Meeting on Control Problems VSPU-2019]. Moscow, 2019, pp. 1439-1443. (in Russian).

[26] Holdsworth B., Woods C. Digital Logic Design. 4th ed. Oxford, Elseiver, 2002. 519 p.

[27] Mandel'shteyn M.L. Avtomaticheskie sistemy upravleniya tekhnologicheskim protsessom bragorektifikatsii [Automatic control systems for the technological process of brews distillation]. Moscow. 1975. 241 p. (in Russian).

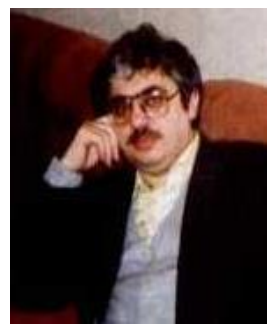

\section{Стопакевич Алексей Аркадьевич,}

кандидат технических наук, доцент, ст. научн. сотр.

Государственный университет «Одесская политехника».

Область научных интересов системный анализ и теория сложных систем управления, многомерные системы управления технологическими процессами промышленности, проблемы кибербезопасности.

Украина, Одесса

E-mail: stopakevich@opu.ua 